\title{
Organización temporal y estrés: un estudio con jóvenes trabajadores del sector turístico en Holguín*
}

\author{
Time management and stress: a study with young workers in the \\ tourism sector in Holguin
}

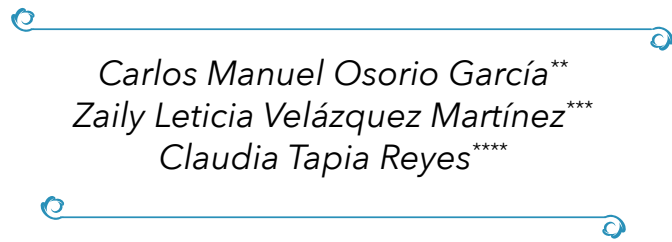

Recibido 07. 05. 2019• Arbitrado 10.06. 2019 • Aprobado 18.06. 2019

* Este artículo presenta los resultados de la investigación titulada: "Estudio sobre la organización temporal en jóvenes trabajadores del turismo que presentan síntomas de estrés". Esta indagación, es un resultado del proyecto Institucional del Departamento de Psicología de la Universidad de Holguín nombrado "Centro de Orientación psicosocial para el desarrollo $\mathrm{Hu}$ mano", con código 2015-37.

** Máster en Trabajo Social, Universidad de Camagüey, Cuba. Departamento de Psicología, Universidad de Holguín, Holguín, Cuba. cmosorio@ uho.edu.cu. ORCID: 0000-0001-86222066.

*** Máster en Dirección, Universidad de Holguín, Cuba. Departamento de Psicología, Universidad de Holguín, Holguín, Cuba. zaily@uho.edu.cu. ORCID: 0000-0002-7378-3128.

**** Licenciada en Psicología, Universidad de Holguín, Cuba. Hotel Brisas Guardalavaca, Banes, Cuba. clau.tapia@nauta.cu

\section{Resumen}

Varias investigaciones con distintos grupos de sujetos vinculan la organización temporal y el estrés como objeto de sus investigaciones. En Cuba son escasas las investigaciones encontradas sobre la problemática, por tanto, profundizar en el estudio de esa relación es el centro de este trabajo. El objetivo del presente estudio es caracterizar la organización temporal en jóvenes trabajadores del sector turístico que presentan síntomas de estrés. La indagación parte de una metodología cualitativa y, el estudio de casos múltiples es la estrategia metodológica implementada con cuatro jóvenes de ambos sexos. Para la recogida de información se utilizaron las siguientes técnicas: entrevista, el inventario de estrés de la vida cotidiana, el pastel y la composición. Las reflexiones teórico metodológicas se realizaron utilizando como unidades de análisis: la organización temporal de la vida como habilidad general del desarrollo personal y el estrés. Los resultados más relevantes apuntan a que los sujetos presentan síntomas de estrés por dificultades en la organización temporal, centrando su tiempo fundamentalmente en el trabajo, a partir 
de una pobre capacidad de reflexión y valoración en cuanto a lo que implica y conlleva la organización temporal de la vida. Se observa que, la organización temporal tiende a ser reproductiva y rutinaria, producto de un proceso de aprendizaje estereotipado en etapas tempranas y a la familiaridad acrítica actual sobre esta habilidad.

Palabras clave: Organización temporal, estrés, habilidad, salud mental, desarrollo humano.

\section{Abstract}

Several researches with different groups of subjects link the time management and stress as object of their researches. In Cuba there are few investigations on the problem, therefore, deepening the study of this relationship is the focus of this work. Therefore, the objective of this study is to characterize the time management of young workers in the tourism sector who present symptoms of stress. The investigation is based on a qualitative methodology and the multiple case study was the methodological strategy implemented with four young people of both sexes. The following techniques were used to collect information: interview, daily life stress inventory, cake and composition. The theoretical methodological reflections were carried out using as units of analysis: the time management in the life as a general skill of personal development and stress. The most relevant results indicate that the subjects present symptoms of stress due to difficulties in the time management, focusing their time fundamentally on work, based on a poor capacity for reflection and assessment as to what the time management of life implies and entails. It was observed that the time management tends to be reproductive and routine, product of a stereotyped learning process in early stages and the current uncritical familiarity about this ability.

Keywords: time management, stress, ability, mental health, human development

\section{Introducción}

El sector turístico en Cuba es un renglón priorizado por los aportes que realiza a la economía del país y al desarrollo del producto interno bruto. Este sector ofrece opciones de empleo a una gran cantidad de jóvenes egresados de distintas formas de estudio, entre ellas: la licenciatura en Turismo, los cursos de las escuelas de hotelería y turismo (FORMATUR) y la enseñanza politécnica básica.

En varios acercamientos realizados por los autores de este trabajo a un grupo de jóvenes trabajadores de un hotel del grupo Cubanacán perteneciente al Ministerio del Turismo de Cuba, se pudo conocer que varios de 
ellos expresaban cansancio y fatiga producto a la acumulación de tensiones que les provocaba tener que cumplir con un estándar de calidad reconocido mundialmente y al escaso tiempo que les quedaba para cumplir con sus tareas, funciones y obligaciones en las demás esferas de su vida cotidiana. Muchos de estos jóvenes tienden a viajar desde lugares distantes hacia el hotel, ejemplo de ello son los que deben recorrer la distancia entre la ciudad de Holguín o desde la ciudad de Banes hasta el polo turístico de Guardalavaca o Pesquero. El cansancio, la fatiga y la acumulación de tensiones pueden estar condicionados por limitaciones en la organización temporal y generar malestar psicológico en estos jóvenes que laboran en la industria turística.

Un desarrollo óptimo de la habilidad organización temporal puede contribuir a que la persona alcance un desempeño eficaz en las distintas esferas de la vida cotidiana, incluida la laboral, tan importante en la juventud. Sin embargo, si las personas no realizan una organización temporal consciente, flexible y crítica, en la que se incluyan cada una de las acciones y operaciones que configuran la habilidad, pueden estar sometidas a un nivel de estrés tan elevado que afecte su salud mental. Esta manifestación puede revelarse también en las personas por estar expuestas a situaciones de elevada exigencia junto a la diversidad de roles, funciones y responsabilidades que deben cumplir y a la inhabilidad para priorizar y romper rutinas de vida.

La influencia de una organización temporal acrítica y rígida puede ser profunda, al punto de provocar distrés en las personas. El distrés, entendido como un estrés perjudicial se revela en "emociones negativas (miedo, depresión, ansiedad, hostilidad), puede obstaculizar nuestro funcionamiento personal, familiar, laboral y/o social e impactar en el sistema neuroendocrino e inmune, modulando ante ciertas condiciones la aparición de disturbios y enfermedades" (Chacón, Grau y Reyes, 2012, p. 20).

Evidencias de estrés por limitaciones en la organización temporal se han identificado en jóvenes estudiantes universitarios en el contexto foráneo. Las investigaciones que reflejan este fenómeno son las de: Misra y McKean (2000); De Sousa, De Oliveira y Clark (2003); Bondan y Bardagi (2008); Costa y Polak (2009); Basso, Graf, Lima, Schmidt y Bardagi (2013) y Pereira, Barbosa, Teixeira, De Oliveira y Rezende (2014). En ellas se considera que las dificultades en la organización temporal son fuente de estrés, pero no se profundiza en las maneras particulares de configuración de la habilidad que afectan el bienestar psicológico y la salud mental de los sujetos.

En el contexto cubano se encontró el trabajo de Peña (2017) que vincula la organización temporal y el estrés. Esta indagación se realiza con maestros de la enseñanza primaria y sirve como referente para el presente trabajo porque, 
no solo identifica que los sujetos sufren estrés por limitaciones en la organización temporal, también profundiza en la configuración de la habilidad, por tanto, refleja las interioridades de la organización temporal que pueden constituir factores estresores.

La organización temporal de la vida se ha estudiado desde 1954 cuando se introdujo el término (Claessens, Van Eerde, Rutte y Roe, 2007). Desde esa fecha son muchos los investigadores interesados en el tema. Los referentes más utilizados son los de Bond y Feather (1988); Macan, Shahani, Dipboye y Philips (1990) y los de Britton y Tesser (1991), según Claessens et al. (2007); Liu et al. (2009) y Ling y Rijmen (2011). Sin embargo, el referente que se asume en esta indagación es el de Graf, Iliasov y Liaudis (1983) por considerarse una posición más completa, en la que se incluyen contenidos que no se consideran en otras propuestas, etc. Estos autores asumen una postura histórico culturalista que resalta el desarrollo dialectico de la habilidad.

La organización temporal de la vida es considerada una habilidad compuesta por cuatro acciones que conforman la estructura interna de la habilidad, estas fueron propuestas por Graf, Iliasov y Liaudis (1983), ellas son: la planificación de sentido, el control de condiciones, la planificación probable y el control de la marcha o final; estas acciones se complementan por las operaciones formuladas por Iliasov (1981), ellas son: utilización de medios especiales, previsión, ajuste del tiempo, priorización, constancia y persistencia, proyección de planes y el control del cumplimiento del plan.

La planificación de sentido "juega el papel de orientación a través de la jerarquía motivacional de la persona, de los sentidos que orientan su vida (sentido de la vida). Estas jerarquías pueden ser establecidas para distintos plazos (largo, mediano y corto)" (Fariñas, 2004, p. 100).

Por otra parte, la función del control de condiciones se describe como "la consideración de las situaciones en que se llevan a cabo las actividades cotidianamente, tanto desde un punto de vista subjetivo como objetivo, lo cual permite una organización más adecuada y realista de la vida" (Fariñas, 2004, p.100).

El pronóstico probable es:

(...) la delimitación del tiempo en que se prevé tengan lugar las diferentes actividades, relaciones y tareas de la vida (fechas, horarios). En otras palabras es una suerte de anticipación flexible, donde puede ser conveniente adelantar, aplazar, cancelar, sustituir, incluir las cosas que se espera hacer, según se vayan presentado los acontecimientos (Fariñas, 2004, p. 101). 
Por último, el control de los resultados se define como: "ese balance que hacemos de lo hecho, con algún grado de frecuencia y nivel de conciencia" (Fariñas, 2004, p. 102).

Las operaciones que conforman la habilidad son definidas por Iliasov (1981, citada en Pérez, 1989), de la siguiente manera:

1. Utilización de medios especiales: apoyo externo de que se vale el sujeto, lo cual resulta necesario debido a la complejidad de la época actual, mediante agendas, libretas de apuntes, hojas, etc.

2. Previsión: Conocimiento anticipado, por parte del sujeto, de lo que él tiene que hacer próxima o lejanamente (día, semana, mes, año).

3. Ajuste del tiempo: Conocimiento aproximado, por parte del individuo, del tiempo que necesita para calcular y realizar las actividades. Ajuste del tiempo sobre la marcha, adelanto o postergación de tareas, etc.

4. Priorización: El sujeto, según la significación de las tareas a cumplir, prioriza las mismas, dándole mayor peso a aquellas que son más importantes, aunque no debe olvidar las de menor urgencia, con el fin de concretar las actividades planificadas y evitar los pendientes.

5. Constancia y persistencia: Frecuencia estable con que el sujeto elabora planes relativos a la organización de su vida. Regularidad del tiempo que el sujeto emplea en la realización de actividades cotidianas, de acuerdo al espectro de intereses, necesidades, gustos, etc. Aquí se incluyen las actividades concretas que el individuo lleva a cabo.

6. Proyección de planes: Alcance que tiene para el sujeto la planificación de su tiempo (en términos de día, semana, mes, año).

7. Control del cumplimiento del plan: control por parte del sujeto, de si se han realizado o no las tareas que se había propuesto cumplimentar.

La organización temporal de la vida influye de manera general en la eficacia del sujeto, en todas las esferas de la vida cotidiana. Particularmente es una habilidad que necesita ser desarrollada de manera tal que contribuya al desempeño eficaz en el ámbito laboral, específicamente en los jóvenes que comienzan su vida laboral, cuando esto no sucede puede constituir un factor estresor para ellos. Ante las muestras de cansancio, fatiga y de acumulación de tensiones que emergieron en varios encuentros con un grupo de jóvenes trabajadores del sector turístico se consideraron como objetivos del estudio: 1. Diagnosticar el estrés cotidiano al que están sometidos un grupo de jóvenes trabajadores del sector turístico y 2. Caracterizar la organización temporal en esos jóvenes que presentan síntomas de estrés. 


\section{Metodología}

La investigación se realizó entre enero y mayo de 2018. En ella se asumió el enfoque cualitativo y como estrategia del diseño el estudio de casos múltiples. La indagación tiene un carácter descriptivo pues lo que se busca es la esencia de cómo es y cómo se manifiesta la organización temporal en jóvenes trabajadores del sector turístico que presentan síntomas de estrés. Se tuvo en cuenta que la selección de los casos se realizara "sobre la base de la potencial información que la rareza, importancia o revelación que cada caso concreto pueda aportar al estudio en su totalidad" (Rodríguez, Gil y García, 2004, p. 96).

La selección de los casos se realizó de manera intencional y propositiva. Para la indagación se tuvieron en cuenta los siguientes criterios: se tenga fácil acceso al campo; exista una alta probabilidad de que se den una mezcla de procesos, programas, personas, interacciones y/o estructuras relacionadas con las cuestiones de investigación; se pueda establecer una buena relación con los informantes; el investigador pueda desarrollar su papel durante todo el tiempo que sea necesario; se asegure la calidad y credibilidad del estudio.

Estos elementos, de acuerdo a su debida interpretación y ejecución, ofrecieron la oportunidad de profundizar acerca del fenómeno en cuestión y los casos evaluados.

Al acceder al campo se solicitó el consentimiento a la dirección del hotel para realizar la indagación. Después se realizó una entrevista al subdirector del departamento de recursos humanos de la entidad en la que se obtuvo información sobre la cantidad de jóvenes con edades entre los 20 y los 35 años que trabajan en el hotel. Una vez solicitado el consentimiento, de los 11 sujetos jóvenes que trabajan en el departamento $A+B$ (bebidas y alimentos) o sea, dependientes de servicio gastronómico y cantineros, solo 7 de ellos ofrecieron su disposición a participar en la indagación.

Para la selección de los casos final se aplicó la técnica nombrada "Inventario de estrés cotidiano" de Brantley, Waggoner, Jones y Rappaport, (1987).

Esta técnica consiste en un cuestionario que reúne contrariedades interpersonales, familiares y laborales, cuya intensidad ha de evaluar el propio sujeto, de acuerdo a un puntaje convencional. Cuenta con 58 ítems que se evalúan del 1 al 7 donde el 7 expresa mayor estrés. El cuestionario tiene una evaluación cuantitativa y una cualitativa que se describen a continuación. Resultado del cuestionario de 0 a 120 puntos usted está relajado. Su estrés es puntual y controlado si alcanza de 121 a 200 puntos. Tiene signos de estrés, pero habitualmente los domina si alcanza de 200 a 300 puntos. Su estado de 
estrés es importante y debe prestarle atención si alcanza 361 o más puntos. Este último intervalo expresa que su estado de estrés es patológico, por tanto, debe consultar con un médico.

La evaluación cualitativa se realizó a partir de la valoración del sujeto sobre los acontecimientos que expresan una relación con la organización temporal. Los acontecimientos generadores de tensión o molestia relacionados con la organización temporal corresponden a los ítems: 3-preocupación por el trabajo pendiente; 4-prisa por acabar el trabajo dentro del plazo; 8-incapacidad para acabar un trabajo; 9-estar desorganizado; 24-olvidar algo; 30-preocuparse por el futuro; 39-ser incapaz de cumplir los planes diarios y 58-llegar tarde al trabajo o a una cita.

El inventario de estrés cotidiano permitió diagnosticar el estrés de la vida cotidiana que sufren un grupo de jóvenes trabajadores en una instalación hotelera en Holguín para de ahí seleccionar los casos que formarían el grupo de estudio.

Los casos seleccionados fueron cuatro en total atendiendo a los criterios de selección y a su disposición a participar de la investigación. El rango de edad fue entre los 26 y 33 años. De ellos tres femeninos y uno masculino.

Una vez identificados los casos que muestran síntomas de estrés en la vida cotidiana por limitaciones en la organización temporal, se aplicaron las técnicas del pastel, la guía de entrevista semiestructurada y la composición. A continuación, se describen de manera breve cada una de ellas.

En la técnica del pastel se les solicita a los sujetos que subdividan un círculo en partes, como si fuera un pastel, se les aclara que los tamaños de los pedazos no tienen que ser iguales, sino que deben responder a la cantidad de tiempo que les dedican a las actividades fundamentales que desarrollan en su vida. De esta manera se pretende observar, en qué emplea el tiempo el sujeto de manera general (Suárez, 2007).

La guía de entrevista semiestructurada se aplicó para obtener información sobre cada uno de los sujetos estudiados. El objetivo de este tipo de entrevista es obtener respuestas libres, vinculadas a ciertas áreas. Con anterioridad se organiza un plan específico, la guía se estructura por temas y cuestiones necesarias para discutirse en un orden más o menos particular (Ratner, 2013), en este caso los temas y cuestiones a medirse fueron las acciones y operaciones relacionados con la organización temporal.

La técnica de la composición se tituló "Cómo organizo mi tiempo" y su objetivo consistió en conocer y profundizar en cómo el sujeto organiza su tiempo. 
Los indicadores para evaluar la composición son los ofrecidos por González y Mitjans (1999, pp. 68-69) que incluyen: el contenido, el vínculo emocional hacia el contenido y la elaboración personal.

Contenido: la atención se centra en el número de categorías utilizadas por el sujeto, sea la amplitud y frecuencia de las categorías o conceptos desarrollados en la explicación.

Vínculo emocional: se refiere al tipo de tono emocional o vivencia que el sujeto asocia a los contenidos que expresa.

Elaboración personal: responde al nivel de individualización que logra un sujeto sobre los contenidos que expresa; es cuando la expresión del sujeto adquiere un carácter personalizado, elaboración personal es sinónimo de implicación, de personalización de la información expresada.

El procedimiento seguido para el análisis de los datos obtenidos fue el propuesto por Rodríguez et al. (2004). Estos autores plantean que, para el análisis de los datos cualitativos, después de la recogida de estos, se ejecutan las siguientes tareas: reducción de los datos, disposición y transformación de los datos y por último obtención y verificación de conclusiones.

\section{Resultados}

En este apartado del artículo se presentan los resultados del inventario de estrés cotidiano, del pastel, la observación, la entrevista y la composición que posibilitan la caracterización de la habilidad organización temporal de la vida cotidiana.

\section{Inventario de estrés cotidiano}

Los resultados de la tabla 1 demuestran la puntuación alcanzada por cada uno de los siete sujetos que brindaron su disposición a participar de la investigación una vez evaluado el inventario de estrés cotidiano.

Tabla1. Puntuación alcanzada por cada sujeto una vez evaluado el inventario de estrés cotidiano.

\begin{tabular}{cccc}
\hline Seudónimo & Sexo & Edad & Valor cuantitativo del inventario \\
\hline Yadira & Femenino & 30 años & 126 puntos \\
Sandra & femenino & 26 años & 106 puntos \\
María & Femenino & 33 años & 132 puntos \\
Gabriel & Masculino & 29 años & 138 puntos
\end{tabular}




\begin{tabular}{cccc}
\hline Seudónimo & Sexo & Edad & Valor cuantitativo del inventario \\
\hline Ángela & Femenino & 29 años & 82 puntos \\
Yelena & Femenino & 30 años & 66 puntos \\
Elizabeth & Femenino & 30 años & 59 puntos \\
\hline
\end{tabular}

Fuente: Propia

Como se puede apreciar en el análisis cuantitativo, tres sujetos mostraron un estrés puntual y controlado, ellos son: Yadira que alcanzó un resultado de 126 puntos, María 132 y Gabriel 138 puntos. Uno de los casos, Sandra, obtuvo un resultado de 106 puntos. Aunque en el caso de Sandra la puntuación corresponde a un estado relajado, cuando se analizaron los ítems 3, 4, 8, 9, $24,30,39$ y 58 que responden a contenidos relacionados con la organización temporal se evidenciaron algunas limitaciones relacionadas con la habilidad. Esta es la razón por la que se incluyó el caso de Sandra en la indagación. Este mismo análisis cualitativo se realizó con los otros tres casos.

El análisis cualitativo posibilitó conocer que para María la preocupación por el trabajo pendiente (ítem 3) fue muy estresante, mientras para Yadira y Sandra fue extremadamente estresante y a Gabriel le causó pánico.

En relación con la prisa por acabar el trabajo dentro del plazo (ítem 4) a los cuatro sujetos les resultó extremadamente estresante.

En cuanto a la incapacidad para acabar un trabajo (ítem 8) para María fue poco estresante, mientras para los otros tres sujetos les resultó muy estresante.

Para María, estar desorganizado (ítem 9) resultó poco estresante, mientras para los otros sujetos resultó muy estresante.

En cuanto al (ítem 24) que se refiere al olvido de algo para Sandra fue un acontecimiento poco estresante, para Yadira y Gabriel fue muy estresante y para María resultó extremadamente estresante.

Por otra parte, en el (ítem 30) que se refiere a preocuparse por el futuro para Sandra fue muy estresante, para Yadira y María resultó extremadamente estresante y a Gabriel le causó pánico.

En relación al (ítem 39) concerniente a ser incapaz de cumplir los planes diarios a Sandra y a María les resultó extremadamente estresante y a Yadira y Gabriel les causó pánico.

En otro sentido en el (ítem 58) referente a llegar tarde al trabajo o a una cita Yadira, Sandra y Gabriel expresaron que les resultó muy estresante y para María fue extremadamente estresante. 
A estos datos hay que agregarle que cuando se les preguntó a los sujetos si existía algún acontecimiento generador de tensión que hubieran experimentado y que no estuviera en la lista Yadira, Sandra y Gabriel expresaron algunos relacionados con la organización temporal. Para Yadira, la escasez de tiempo para dedicarle a su familia es un acontecimiento extremadamente estresante. Sandra expresó que tener poco tiempo para su entrenamiento y no poder planificarse otras cosas por el trabajo les resultan muy estresante. Por su parte, Gabriel indicó que el trabajo le ocupó mucho tiempo y que cuando está en la casa se siente muy agotado, estos acontecimientos los clasificó como extremadamente estresante.

\section{Habilidad organización temporal de la vida}

En este apartado se tratarán los resultados que caracterizan la habilidad organización temporal de la vida cotidiana de los jóvenes trabajadores del sector turístico que presentan síntomas de estrés cotidiano.

\section{Planificación de sentido}

La planificación de sentido se concreta en las actividades que realizan los sujetos de manera sistemática, así como las actividades urgentes e imprevistas. En la gráfica que se presenta a continuación se representan las actividades a las que los sujetos le dedican más tiempo.

Gráfico 1. Actividades a las que los sujetos estudiados le dedican más tiempo.

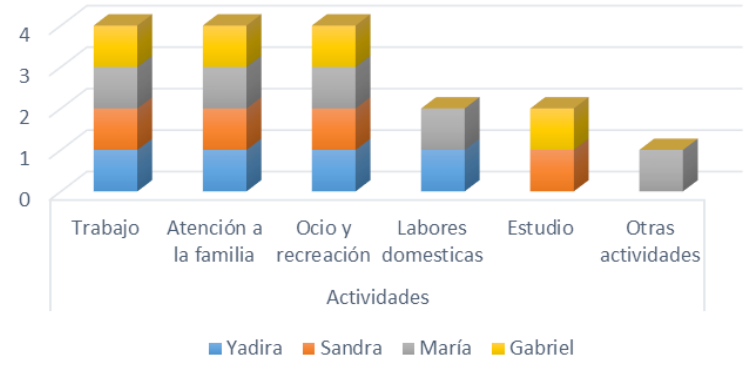

Fuente: Propia

Los cuatro sujetos dedican la mayor parte de su tiempo en el trabajo. En relación a la atención a la familia de procedencia los cuatro sujetos emplean su tiempo en esta actividad, aunque Yadira expresó que también le dedica tiempo a la atención a su esposo. 
En cuanto al ocio y la recreación cada sujeto tiene diferentes maneras de realizarlos, por ejemplo, a Yadira le gusta ver televisión y la computación, además de pasear con su familia; a Sandra le gusta ver televisión, salir a pasear y bailar; María prefiere salir a pasear, las fiestas, bailar y ver televisión; por su parte, a Gabriel le interesa ver televisión, salir a fiestas y jugar en la computadora. En las labores domésticas solo emplean su tiempo Yadira y María. Al estudio le dedican tiempo Sandra que realiza cursos diversos sobre la atención en bares y cantinas y Gabriel que cursa la carrera universitaria de Ingeniería Industrial. Por último, María expresó que les dedica tiempo a las gestiones que tiene que realizar para la construcción de su casa.

Las actividades representadas en la gráfica corresponden a la planificación de sentido que realizan los sujetos, estas juegan un papel central en la priorización que establecen los mismos según sus necesidades, intereses y motivaciones, aunque no se deben olvidar las otras tareas que no son tan sistemáticas y las actividades urgentes que también influyen en la priorización y la planificación de sentido.

\section{Control de condiciones}

En este aspecto se identificó que un sujeto casi nunca tiene en cuenta las condiciones, otro sujeto casi siempre las tiene en consideración mientras, los otros dos solo a veces las consideran. Es necesario resaltar que los cuatro sujetos expresaron tener en consideración solo condiciones materiales. Yadira expresó "yo casi nunca tengo en cuenta las condiciones que necesito para realizar las tareas y cuando lo hago me fijo solamente en las condiciones objetivas, mínimas", Sandra refirió "casi siempre tengo en cuenta las condiciones, por ejemplo para el trabajo me fijo en los recursos que necesito para atender a los clientes, etc.", mientras María indicó "Yo, solo a veces, muy escasas, tengo en cuenta las condiciones que necesito para realizar las tareas, cuando realizo las labores del hogar trato de tener todos los recursos que necesito para limpiar, ..." y Gabriel afirmó "Solo a veces considero las condiciones necesarias para realizar las tareas, a veces considero el local, libros, libretas, entre otras".

\section{Pronostico probable}

\section{Constancia y persistencia}

En relación a la constancia y persistencia se identificaron dos sujetos que no son constantes ni persistentes y los otros dos son constantes a corto plazo, es decir, que solo se planifican a diario o semanal. 
Yadira expresó "Yo no acostumbro a planificar mis actividades, las realizo como van apareciendo" y Gabriel refirió "no planifico mis actividades, soy más espontáneo". Por su parte Sandra relató "Yo me planifico a diario, me parece más práctico", mientras María relató "Mi planificación la realizo semanal, me gusta hacerlo así".

\section{Previsión}

En este punto se puede dividir a los sujetos en previsores a corto, mediano y largo plazos teniendo en cuenta el conocimiento que tienen de lo que deben realizar en el día, la semana, el mes y/o el año. El sujeto previsor a corto plazo es María que afirmó "conozco lo que haré en el día y la semana, pero nunca lo que haré en el mes ni en el año". Los sujetos previsores a mediano plazo son Yadira y Gabriel, la primera alegó "si, conozco lo que tengo que hacer en el día, la semana y el mes, pero no lo que debo hacer en el año" y Gabriel manifestó "Yo no sé lo que haré en el año casi nunca, pero si conozco lo que haré en el día siguiente, la semana y el mes". Por último, el sujeto previsor a largo plazo es Sandra que afirmó "Casi siempre conozco lo que haré todos los días, la semana, el mes y el año".

\section{Proyección de planes}

En relación a la elaboración de planes los sujetos se pueden clasificar como: sujetos que no acostumbran a proyectar planes, sujetos que proyectan planes a corto plazo y los que lo hacen a mediano plazo.

No acostumbra a proyectarse planes Yadira. Este sujeto expresó "no tengo objetivos en la vida sobre los que haya meditado" "tampoco elaboro planes para el día, la semana, el mes, ni el año". Otro sujeto que no acostumbra a proyectarse planes es Sandra. Ella narró que sus objetivos son: "terminar los cursos en los que estoy matriculada" "crecer profesionalmente" y "formar una familia", sin embargo, "casi nunca elaboro planes para el día, la semana, el mes, ni el año".

Por su parte, Gabriel proyecta los planes a corto plazo. Para este sujeto sus objetivos son: "mantener su puesto de trabajo" y "concluir mis estudios universitarios". También afirmó "solo a veces elaboro planes para el día y la semana y casi nunca lo hago para el mes ni para el año".

Por último, María proyecta planes a mediano plazo, es decir, se proyecta el día, la semana y el mes. Este sujeto tiene como objetivos: "Mejorar mis condiciones de vida" y la "Educación de mis hijos", además afirmó "a veces elaboro planes para el día, la semana y el mes", pero "casi nunca me proyecto planes para el año". 
Realización de ajustes

María y Gabriel expresaron que casi nunca realizan ajustes que les permitan adelantar y/o postergar algunas actividades y que solo a veces recuperan el tiempo perdido o utilizado en tareas adelantadas o imprevistas. Sandra y María expresaron que a veces realizan ajustes que les permitan adelantar y/o postergar algunas actividades y que en ocasiones recuperan el tiempo perdido o utilizado en tareas adelantadas o imprevistas. De esta manera se evidencian dificultades en los cuatro casos para realizar ajustes que posibiliten una priorización de actividades, además de limitaciones para cumplir las actividades postergadas, etc.

Uso de medios

Ninguno de los casos utiliza algún medio para planificarse ni para controlar el cumplimiento de sus actividades. Yadira expresó "No utilizo ningún medio porque no lo veo como algo que me pueda ayudar"; Sandra afirmó "No, no he considerado esa idea, tampoco lo veo necesario"; por su parte María dijo "No lo he pensado necesario" y Gabriel afirmó "No lo utilizó y no me parece necesario".

\section{Control de la marcha o los resultados}

En relación al control de condiciones, tres de los sujetos no realizan un control consciente ni sistemático y el otro lo realiza una vez terminadas las actividades, pero tampoco impresiona ser sistemático. Yadira expresó "yo no acostumbro a controlar el cumplimiento de las actividades", Sandra afirmó "No acostumbro a controlar si cumplí o no sencillamente las realizo y ya" por su parte María dijo "Realmente no control si cumplo o no lo planificado. Trato de garantizar el cumplimiento de las tareas más importantes". Por último, Gabriel aseveró "No lo controlo, simplemente cuando lo finalizo o sucede, valoro si tuvieron el resultado que yo esperaba"

\section{Discusión y conclusiones}

A partir del análisis cuantitativo y cualitativo del inventario de estrés cotidiano se pudo conocer que de los siete sujetos que ofrecieron su disposición a participar en la investigación cuatro presentan síntomas de estrés.

Los jóvenes trabajadores del sector turístico emplean el tiempo en mayor medida en la esfera laboral, dedican gran parte de su tiempo a estudios, superación profesional o a su familia y a la recreación. En el trabajo de Peña (2017) los tres maestros que participaron de la investigación emplean el tiempo generalmente en la esfera laboral, además, dedican la mayor parte de su tiempo a la familia y en menor medida a la recreación. 
En relación al control de condiciones para ejecutar las actividades con calidad en el tiempo previsto, uno de los sujetos casi nunca las tiene en cuenta, otros dos solo a veces las consideran y el otro sujeto casi siempre las tiene en consideración. Por su parte, Peña (2017) identificó que los tres sujetos de su indagación no tenían en cuenta las condiciones para la realización efectiva de sus actividades o tareas.

En cuanto al pronóstico probable dos sujetos no acostumbran a planificar las actividades y otros dos las planifican a corto plazo. En Peña (2017) se identifican dos sujetos como no previsores, que no acostumbran a planificar las actividades y el otro planifica las actividades a corto plazo.

En este estudio los sujetos expresaron no utilizar ningún medio para planificar ni controlar sus actividades, sin embargo, en el trabajo de Peña (2017) los sujetos afirmaron que utilizan medios como el plan de trabajo, pero lo ven como algo formal, uno de los sujetos utiliza un diario, pero no para planificar ni controlar la marcha de sus actividades sino para reflejar lo que hizo en el día.

En otro sentido y en relación al control de la marcha y los resultados, tres de los sujetos estudiados no realizan un control consciente ni sistemático y el otro realiza el control una vez finalizadas las actividades, pero esta acción no la realiza de manera sistemática. En el estudio de Peña (2017) ningún sujeto realiza un balance de la marcha, pero si existen indicios de que realizan un balance final de algunas actividades, las más importantes para cada sujeto.

Por último, los sujetos expresaron no haber participado en ningún entrenamiento o programa de intervención dirigido a favorecer el desarrollo de la organización temporal, al igual que en el trabajo de Peña (2017). Este elemento es una de las razones por las que, como tendencia, la organización temporal en los sujetos estudiados se revela como reproductiva y rutinaria, esto puede ser también producto a un proceso de aprendizaje estereotipado desde etapas tempranas y a la familiaridad acrítica actual sobre esta habilidad (Fariñas, 2004; Osorio, Velázquez, y Bringuez, 2017; Osorio, Velázquez, y Rodríguez, 2017, Osorio, 2019).

\section{Agradecimientos}

A los jóvenes trabajadores del hotel del grupo Cubanacán perteneciente al Ministerio del Turismo de Cuba que participaron de la indagación. 


\section{Referencias}

Basso, C., Graf, L., Lima, F., Schmidt, B., Bardagi, M. (2013). Organização de tempo e métodos de estudo: Oficinas com estudantes universitários. Revista Brasileira de Orientação Profissional, 14(2), 277-288. Recuperado de: http://pepsic.bvsalud. org/scielo.php?script $=$ sci_arttext $\&$ pid $=$ S1679-33902013000200012

Bond, M., Feather, N. (1988). Some correlates of structure and purpose in the use of time. Journal of Personality and Social Psychology, 55(2), 321-329. Recuperado de: http://dx.doi.org/10.1037//0022-3514.55.2.321

Bondan, A., Bardagi, M. (2008). Comprometimento profissional e estressores percebidos por graduandos regulares e tecnológicos. Paidéia, 18(41), 581-590. Recuperado de: http://www.scielo.br/scielo.php?pid=S0103863X2008000300013\&script $=$ sci_abstract\&tlng $=$ pt

Brantley, P., Waggoner, C., Jones, G., Rappaport, N. (1987). A Daily stress inventory: development, reliability, and validity. Journal of Behavioral Medicine, 10, 61-74. Recuperado de https://www.ncbi.nlm.nih.gov/pubmed/3586002

Britton, B. K., Tesser, A. (1991). Effects of time-management practices on college grades. Journal of Educational Psychology, 83, 405-410. Recuperado de: https:// www.researchgate.net/profile/Abraham_Tesser/publication/232573913 Effects_of_Time-Management_Practices_on_College_Grades/ links/0046353545613de89d000000/Effects-of-Time-Management-Practices-onCollege-Grades.pdf

Claessen, B., Van Eerde, W., Rutte, C.G., Roe, R.A. (2007). A review of the time management literature. Personnel Review, 36(2), 255-276. Doi $10.1108 / 00483480710726136$

Costa, A., Polak, C. (2009). Construção e Validação de Instrumento para Avaliação de Estresse em Estudantes de Enfermagem. Rev. Esc. Enferm. USP, 43 (Esp.), 1017-1026. Recuperado de: http://www.scielo.br/scielo.php?script=sci_ arttext\&pid $=$ S0080-62342009000500005

Chacón, M., Grau, J., Reyes, M. (2012). Prevención y control del stress laboral y el desgaste. Guía para profesionales que trabajan con enfermos crónicos avanzados. La Habana: Editorial Ciencias Médicas.

De Sousa, E., De Oliveira, E., Clark, C. (2003). Avaliação do estresse e das habilidades sociais na experiência acadêmica de estudantes de medicina de uma universidade do Rio de Janeiro. Interação em Psicologia, 7(2), 43-51. Recuperado de: http:// www.scielo.br/scielo.php? pid=S1414-98932016000100224\&script $=$ sci abstract\&tlng $=$ pt. 
Fariñas, G. (2004). Maestro, para una didáctica del aprender a aprender. La Habana: Pueblo y Educación.

Graf B., Liaudis V. Ya., Iliasov I.I. (1983). Bases para la organización de la actividad de estudio y el trabajo independiente de los alumnos. Moscú: Editorial M.G.Y.

González, F., Mitjans, A. (1999). La personalidad: su educación y desarrollo. La Habana: Editorial Pueblo y Educación. (3ra edición).

Iliasov, J. J. (1981). Fundamentos para la autoorganización de la actividad docente y del trabajo independiente de los alumnos. Moscú: Editorial de la Universidad de Moscú.

Liu, O., Rijmen, F., MacCann, C., Roberts, R. (2009). The assessment of time management in middle-school students. Personality and Individual Differences, 47(3), 174-179. Recuperado de: http://dx.doi.org/10.1016/j.paid.2009.02.018.

Ling, G. y Rijmen, F. (2011). A General Procedure to Assess the Internal Structure of a Noncognitive Measure-The Student 360 Insight Program (S360) Time Management Scale. ETS Research Report Series, 2011(2), i-37. http://dx.doi. org/10.1002/j.2333-8504.2011.tb02278.x

Macan, T.H., Shahani, C., Dipboye, R.L., Philips, A.P. (1990). College students' time management: correlations with academic performance and stress. Journal of Educational Psychology, 82, 760-768. Recuperado de: https://www.google.com.cu/ url sa $=\mathrm{t} \& \mathrm{rct}=\mathrm{j} \& \mathrm{q}=\& \mathrm{esrc}=\mathrm{s} \&$ source $=$ web\&cd $=1 \& \mathrm{cad}=$ rja\&uact $=8 \& \mathrm{ved}=2 \mathrm{ahUKE}-$ wif_pbWpLfhAhUmuVkKHXAmAGoQFjAAegQIAhAC\&url=https\%3A\%2F\%2Fpdfs. semanticscholar.org\%2Fea72\%2F13c01261e9e172f1c362be3781df30f6f5b7.pdf\&us$\mathrm{g}=\mathrm{AOvVaw0GrcfjNoXFgAK8S5ZRWD-4}$

Misra, R., McKean, M. (2000). College student's academic stress and its relation to their anxiety, time management and leisure satisfaction. American Journal of Health Studies, 16, 41 -51. Recuperado de: https://www.researchgate.net/publication/209835950 College_students\%27academic_stress_and_its_relation_to_their_anxiety_time_ management_and_leisure_satisfaction

Osorio, C. (2019). Estudio de las configuraciones de la organización temporal en estudiantes de psicología. Informe de investigación. Holguín, Cuba: Universidad de Holguín, Facultad de Ciencias Sociales, Departamento de Psicología.

Osorio, C., Velázquez, Z. y Bringuez, M. (2017). Organización del tiempo y proyecto de vida en jóvenes instructores de arte. En VIII Conferencia Científica Internacional de la Universidad de Holguín, Cuba. ISBN: 978-959-16-3272-2

Osorio, C., Velázquez, Z., Rodríguez, Y. (2017). La organización del tiempo en jóvenes cuentapropistas. Reflexiones para un análisis desde la perspectiva de género. En II 
Convención Internacional de Ciencias Sociales y Ambientales. Universidad de Santiago de Cuba, Cuba. ISBN: ISBN.978-959-207-577-1

Peña, Y. (2017). Estudio de la organización del tiempo en maestros jóvenes que presentan síntomas de estrés. (Tesis de licenciatura inédita). Universidad de Holguín, Holguín, Cuba.

Pereira, M., Barbosa, M., Teixeira, R., de Oliveira, E., Rezende, J. (2014). Sofrimento Psíquico. Percepção dos estudantes de medicina e a forma como lidam com a situação. 3er Congreso Ibero-americano de Investigación Cualitativa. Volumen 3. pp. 160-167. Recuperado de: https://proceedings.ciaiq.org/index.php/CIAIQ/ article/view/456.

Pérez, R. (1989). Estudio acerca de la perspectiva temporal futura en una muestra de jóvenes preuniversitarios cubanos. (Tesis de licenciatura inédita). Universidad de La Habana, La Habana, Cuba.

Ratner, C. (2013). Desde Vygotski a la Psicología Macrocultural. Obras escogidas. España: Documenta Universitaria.

Rodríguez, G., Gil, J., García, E. (2004). Metodología de la investigación cualitativa. La Habana: Editorial Félix Varela.

Suárez, L. (2007). Un acercamiento al desempeño profesional de los maestros de la enseñanza primaria. (Tesis de licenciatura inédita). Universidad de la Habana, La Habana, Cuba. 